\title{
Technè
}

La science au service de l'histoire de l'art et de la préservation des biens culturels

47 | 2019

Bernard Palissy : nouveaux regards sur la céramique française auX $\mathrm{XVI}{ }^{e}$ et $\mathrm{XVII}$ siècles

\section{État de la bibliographie depuis 2009}

Bibliographical update since 2009

\section{Vincent Rousseau}

\section{OpenEdition}

Journals

Édition électronique

URL : http://journals.openedition.org/techne/1616

DOI : $10.4000 /$ techne. 1616

ISSN : 2534-5168

Éditeur

C2RMF

\section{Édition imprimée}

Date de publication : 1 juin 2019

Pagination : 140-142

ISBN : 978-2-11-152830-7

ISSN : $1254-7867$

Référence électronique

Vincent Rousseau, «État de la bibliographie depuis 2009 », Technè [En ligne], 47 | 2019, mis en ligne le 01 juin 2019, consulté le 14 septembre 2020. URL : http://journals.openedition.org/techne/1616

\section{(c) (†) $\ominus$}

La revue Technè. La science au service de l'histoire de l'art et de la préservation des biens culturels est mise à disposition selon les termes de la Licence Creative Commons Attribution - Pas d'Utilisation Commerciale - Pas de Modification 4.0 International. 
140 La bibliographie de référence sur Bernard Palissy reste celle rédigée par Pierre-Jean Trombetta publiée aux pages 207 à 234 de l'ouvrage suivant : Lestringant F. (dir.), 1992, Bernard Palissy (1510-1590), l'Écrivain, le Réformé, le Céramiste, actes du colloque Bernard Palissy, 29-30 juin 1990, Saintes, Abbayeaux-Dames, éditions InterUniversitaires, Mont-de-Marsan.
Elle est reprise et complétée en 2010 dans une nouvelle impression de l'ouvrage diffusée par la librairie Honoré Champion.

Nous mentionnons donc ici uniquement les textes publiés depuis 2009 ou ceux antérieurs à cette date mais absents du travail de Pierre-Jean Trombetta.

\section{Références jusqu'à 2009}

Babelon J.-P., 1989, « Grotte de Verneuil », dans Babelon J.-P., Châteaux de France au siècle de la Renaissance, Flammarion, Picard, Paris, p. 595-596.

Bouquillon A., 2004, "Heaven and earth, 'Madone col Bambino' and 'Rustiques figulines'”, Applied Physics A, vol. 79 $\mathrm{n}^{\circ} 2$, p. 161-166.

Bouquillon A., Leconte O., 2004, « Relire Palissy à la lumière des analyses », Technè, n 20, p. 69-76.

Bress C., 1974, "Palissy and the fiery furnace", Art and antiques weekly, vol. 16 , $\mathrm{n}^{\circ} 2$, p. 20-23.

Clémens J., 1991, «Bernard Palissy : étude de personnalité », dans Les lettres au temps de Henri $I V$, actes du $4^{\mathrm{e}}$ colloque de l'association Henri IV, Agen-Nérac, 18-20 mai 1990, Association Henri IV, Pau, p. 309-327.

Crépin-Leblond T., 1997, « L'exposition du musée d'Écouen : la terre vernissée de Bernard Palissy et la céramique de Saint-Porchaire ", L'Estampille, l'Objet d'art, $\mathrm{n}^{\circ} 318$, p. 68-69.

Espagnet F., 1987, " Palissy aux Tuileries ", La Revue de la céramique et du verre, $\mathrm{n}^{\circ} 32$, p. $12-15$.

Feller C., 2007, « Une fausse rupture ou l'intérêt du retour aux sources en histoire de l'agronomie : l'exemple de la nutrition minérale des plantes et du "génial" Palissy ", dans Robin P., Aeschlimann J.-P., Feller C. (dir.),
Histoire et agronomie, entre ruptures et durée, actes du colloque, Montpellier, 20-22 octobre 2004, IRD éditions, Paris, p. 181-201.

Garnier É., 1881, «Collections de M. Spitzer, céramique française (deuxième et dernier article) : terres émaillées de Bernard Palissy ", Gazette des beaux-arts, tome 24 , p. 487-495.

Guth P., 1953, « Le véritable secret de Palissy ", Connaissance des arts, vol. 1, $\mathrm{n}^{\circ} 15$, p. 52-57.

Havard H., 1894, " Palissy et ses rustiques figulines ", dans Havard H., Les arts de l'ameublement. 2, la céramique, Charles Delagrave, Paris, p. 72-76.

Heuer A., Bouquillon A., Castaing J., 2008 , Bernard Palissy (1510-1590): materials Engineer "Par excellence ", Art Institute of Chicago, Northwestern University Symposium : "Productive affinities: successful collaborations between Museums and Academia”, 29-31 October 2008.

Jacqmin J., 2006, « Étude et restauration d'un plat attribué à Bernard Palissy », Patrimoines, $\mathrm{n}^{\circ}$ 2, p. 111-117.

Karjakina T., 1981, "Palissy atelier ceramics in the Kuskovo collection", Dekorationoe iskusstvo SSSR, n² 286, p. 46-47.

Katz M. P. , Lehr R., 1996, «Quand l'esprit de Palissy soufflait en Touraine ", Connaissance des arts, $\mathrm{n}^{\circ} 529$, p. 82-89.

Kayser P., 2006, "The intellectual and the artisan: Wenzel Jamnitzer and Bernard Palissy uncover the secrets of nature", Australian and New Zealand Journal of art, vol. $7, \mathrm{n}^{\circ} 2$, p. 45-61.
Kemp M., 1997, "Palissy's Philosophical Pots", Nature, $390: 5$.

Kemp M., 1999, "Palissy's Philosophical Pots. Ceramics, Grottoes and the 'Matrice' of the Earth", dans Tega W. (dir.), Le origini della modernità, vol. 2, Olski, Florence, p. 69-89.

Labarte J., 1872, «Faïence émaillée de Bernard Palissy ", dans Histoire des arts industriels au Moyen-Âge et à l'époque de la Renaissance, tome III, chapitre IV, Veuve A. Morel et Cie, Paris, p. 349-358.

Lahaussois C., 1990, «Bernard Palissy : la réalité dépasse le mythe ", L'industrie céramique, $\mathrm{n}^{\circ} 853$, p. 677-680.

Lehr R., 1991, "Les émules de Palissy ", L'Estampille, l'objet d'art, n 249, p. 60-67.

Lestringant F., 2009, «Bernard Palissy ou l'inquiétante étrangeté, le "potier du roi" en son demi-millénaire ", Bulletin de la société de l'histoire du protestantisme francais, tome 155 , p. 767-779.

Malmenaide I., 1997, «Les suiveurs de Bernard Palissy ", France-antiquités, $\mathrm{n}^{\circ} 89$, p. 17-27.

Migeon G., 1896, « Bernard Palissy ou SaintPorchaire ", Gazette des beaux-arts, tome 15, p. 382-387.

Morley J., 1962, "Palissy and the English", Apollo, vol. 77, n 10, p. 794-796.

Miller N., 1977, "Palissy, schemes for grottos and 'rochers': Chenonceau, Tuileries", dans Miller N., French Renaissance foutains, Garland, coll. Outstanding dissertations in the fine arts, New York, p. 254-265. 
Palissy B., 2000, Discours admirables de la nature des eaux $\mathcal{E}$ fontaines tant naturelles qu'artificielles, des métaux, des sels $\mathcal{E}^{\circ}$ salines, des pierres, des terres, du feu $\mathcal{E} \sigma^{\circ}$ des émaux..., Paleo, collection Classique de l'histoire des sciences, ClermontFerrand.

Plaziat J.-C., 1990, « Les fossiles du Tertiaire parisien dans l'œuvre scientifique et artistique de Bernard Palissy (15101590), en commémoration du quatrième centenaire de sa mort ", Travaux du Comité français d'histoire de la géologie, COFRHIGEO, $3^{\mathrm{e}}$ série, tome IV, $\mathrm{n}^{\circ} 8$, p. $78-84$.

Plaziat J.-C., 1997, « L'importance des coquilles fossiles du Tertiaire parisien dans l'œuvre scientifique et artistique de Bernard Palissy, à la fin du seizième siècle », dans Gohau G. (éd.), De la géologie à son histoire, ouvrage édité en hommage à François Ellenberger, Comité des Travaux Historiques et Scientifiques, Paris, p. 15-24.

Plaziat J.-C., 2006, « Bernard Palissy (1580), premier interprète des fossiles de la montagne de Reims : l'énigme du Lutétien de "Venteul au pays de Valois" enfin résolue ", Bulletin d'information des géologues du Bassin de Paris, $\mathrm{n}^{\circ} 43-2$, p. 23-31.

Plaziat J.-C., 2009, « Bernard Palissy à Venteuil en Champagne : un épisode essentiel d'une biographie démythifiée. La montagne de Reims depuis l'origine de la paléontologie et de la géologie françaises (1580-2007) ", Études marnaises (Bull. de la SACSAM), tome 124, p. 53-111.

Poulain D., 1993, « Les "rustiques figulines" du musée des Beaux-Arts de Lyon ", Bulletin des Musées et Monuments lyonnais, vol. 3-4, p. 24-27.

Perrin I., Velde B., Mac Arthur D., 1997, "Les glaçures de Bernard Palissy : une technique originale d'opacification ", Technè, $\mathrm{n}^{\circ}$ 6, p. 58-64.

Perrin I., 1999, « L'art du pastiche dans la céramique du XIx ${ }^{\mathrm{e}}$ siècle ", Musée des arts et métiers, la revue, $\mathrm{n}^{\circ} 26$, p. 21-27.

Schweizer S., 2008, « Die Gartenkunst des Bernard Palissy ", dans Cat. Exp. Benrath, 2008: Wunder und Wissenschaft : Salomon de Caus und die Automatenkunst in den Gärten um 1600 [Exp. Benrath, Museum für europäische Gartenkunst, Stiftung Schloss und Park Benrath, 2008], Grupello, Düsseldorf, p. 198-199.

Slitine F., 1997, « La céramique française de la Renaissance : sa redécouverte au XIX siècle ", L'Estampille, l'Objet d'art, $\mathrm{n}^{\circ} 318$, p. 56-67.

Tite M., 1996, "Comparative study of the production technology for 'SaintPorchaire' and related European ceramics", in Barbour D., Sturman S. (eds.), Saint-Porchaire Ceramics, Studies in the History of Art 52, Monograph Series II, National Gallery of Art, Washington D.C., p. 99-106.

Trombetta P.-J., Poulain D., 1998, « Bernard Palissy, le retour », dans Cat. Exp.
Guiry-en-Vexin, 1998 : Aspects méconnus de la Renaissance en Ille-de-France [Exp. Guiry-en-Vexin, musée archéologique du Val-d'Oise, 1998-1999], Somogy, Paris, p. 238-249.

Willesme J.-P., 1999, « La grotte de Bernard Palissy d'après les fragments conservés au musée Carnavalet », Bulletin de la société de l'histoire de Paris et de l'île-deFrance, p. 89-105.

\section{Documents inédits}

David S., 2003, La collection de céramique issue des fouilles de l'atelier de Bernard Palissy au Louvre, synthèse historique des trouvailles et des restaurations exécutées jusqu'à ce jour, suivi de la campagne actuelle de restauration en vue d'une ouverture de salle au musée d'Écouen, mémoire d'étude (M1), École du Louvre, Paris.

Fernbach I. N., 2006, Beyond pastoral: rusticity and the reframing of court culture in 16th century culture French literature, thèse, université de Californie, Berkeley.

\section{Références depuis 2009}

Andrews N., 2015, "The space of knowledge: artisanal epistemology and Bernard Palissy", Res: Anthropolgy and Aesthetic, vol. 65-66, p. 275-288.

Bailly M., Bouquillon A., Durey V., Roisine G., 2017, «Les émaux de Bernard Palissy à l'épreuve de l'archéologie expérimentale ", Patrimoines : revue de l'Institut national du patrimoine, $\mathrm{n}^{\circ} 13$, p. 72-79.

Barbe F., 2010, Recension de l'article de Jean-Claude Plaziat, "Bernard Palissy, coquillages ou fossiles : du nouveau sur les "rustiques figulines" ", Bulletin monumental de la Société Francaise d'Archéologie, n 168-3, p. 29-30.

Barbe F., Bouquillon A., 2010, « À la redécouverte des créations de Bernard Palissy : l'aiguière et le plat à décor de "rustiques figulines" du musée du Louvre ", La Revue des musées de France, Revue du Louvre, n 5, p. 24-33.

Beentjes T., Smith P. H., 2013, "Sixteenthcentury life casting techniques: experimental reconstructions based on a preserved manuscript", dans Saunders D., Spring M., Meek A., The Renaissance Workshop, Archetype, British Museum, Londres, p. 144-151.

Bergbauer B., Gerbier A., 2013, «Bernard Palissy et l'orfèvrerie de terre ", dans Cat. Exp. Poitiers, 2013 : La licorne et le bézoard, une histoire des cabinets de curiosités [Exp. Poitiers, musée SainteCroix et espace Mendès-France, 20132014], Gourcuff Gradenigo, Montreuil, p. 139.

Bouquillon A., Barbe F., Lehuédé P. et al., 2013, "Bernard Palissy: scientist and potter of the Renaissance in France", dans Saunders D., Spring M., Meek A. (eds.), The Renaissance Workshop: The Materials and Techniques of Renaissance Art, Archetype Publications in association with the British Museum, London, p. 152-159.
Bouquillon A., Castaing J., Barbe F. et al., 2017, "Lead-glazed Rustiques Figulines (Rustic Ceramics) of Bernard Palissy (1510-1590) and his followers", Archaeometry, vol. 59, issue 1, p. 69-83.

Bouquillon A., Castaing J. , Barbe F. et al., 2018, "French Decorative Ceramics Mass-Produced during and after the 17th Century: Chemical Analyses of the Glazes", Archaeometry, vol. 60, p. 946-965, doi.org/10.1111/arcm.12349.

Bourdon Fernbach I., 2015, « Le paysage de la Réforme en Saintonge : l'exemple de Bernard Palissy », dans Brunet S., Martin P. (dir.), Paysage et religion, perceptions et créations chrétiennes, Comité des Travaux Historiques et Scientifiques, collection Histoire, $n^{\circ} 59$, Paris, p. 19-33.

Brady S., 2010, "Organic Ceramic: from Bernard Palissy in the 16th century to today's artists, the forms of nature have inspired great works of porcelain", Art $\mathcal{E}$ Antiques, vol. 33, n 9, p. 72-83.

Cortade L., 2017, « Bernard Palissy et la théorie du cinéma ", French Review, vol. $90, n^{\circ} 3$, p. 115-124.

Delord J., 2012, « Bernard Palissy, un écologiste au temps de la Renaissance ", La Nature et son souci: philosophie et écologie, no 21 , p. 27-38.

Demonet M.-L., 2016, « Style rustique et "figulines" littéraires : Rabelais, Palissy, Montaigne », comunication présentée dans le cadre du colloque Vices de style et défauts esthétiques (mai 2014, ParisSorbonne), Barbafieri C. et Vialleton J.-Y. (dir.), accessible en ligne sur HAL $<$ halshs-01305913>

Dittmann R., 2016, Naturerkenntnis und Kunstschaffen : Die Discours admirables von Bernard Palissy, Übersetzung und Kommentar, De Gruyter, Berlin.

Dupuis J., 2016, "La céramique dite "d'Avon" : retour historiographique et mise au jour d'une attribution légendaire ", Revue de l'Art, n 193/20163 , p. $27-34$

Duveen J. H., 2013, "The Fatal Plate of Bernard Palissy", dans Secrets of an art dealer, Read Books limited, Redditch.

Felfe R., 2015, Naturform und bildnerische Prozesse, Elemente einer Wissensgeschichte in des Kunst des 16. und 17. Jahrhunderts, De Gruyter, collection Actus et Imago, vol. 13, Berlin.

Feller C., Aeschlimann J.-P., 2014, "Soil and salts in Bernard Palissy's (1510-1590) view: Was he the pioneer of the mineral theory of plant nutrition ?" dans Churchman G. J., Landa E. (dir.), The soil underfoot: infinite possibilities for a finite resource, CRC Press, Taylor \& Francis Group, Boca Raton, FL, p. 289299.

Ferdinand J., 2016, « Placere et docere : le jardin minéral de Bernard Palissy ", dans Ferdinand J. (dir.), From art to science: experiencing nature in the European garden, 1500-1700, ZeL Edizioni, coll. Festina lente Mescellanea, $n^{\circ}$ 4, Treviso, p. 92-109. 
Fernbach I., 2010, L'invention du discours rustique dans la littérature française $d u$ $X V I^{e}$ siècle, Peter Lang (dir.), coll. Currents in comparative romance languages and literatures, vol. 170, New York.

Gerbier A., 2013, " "Les choses admirables et monstrueuses" de Bernard Palissy ", dans Cat. Exp. Poitiers, 2013 : La licorne et le bézoard, une histoire des cabinets de curiosité [Exp. Poitiers, musée SainteCroix et espace Mendès-France, 20132014], Gourcuff Gradenigo, Montreuil, p. $136-138$

Hermann M., 2010, «Bernard Palissy, céramiste précurseur », Collectionneur et chineur, $\mathrm{n}^{\circ} 82$, p. 47.

Huchon M., 2010, « Palissy et un monde journellement renouvelé : rêverie sur le psaume 104 et les coquilles ", dans Thomasset C., Ducos J., Chambon J.-P., Aux origines de la géologie de l'Antiquité au Moyen Âge, actes du colloque international, 10-12 mars 2005, Paris Sorbonne (Paris IV), Honoré Champion, Paris, p. 267-279.

Laube S., 2010, «Wissenswelten sinnlicher Frömmigkeit. Theatral

Antriebsmomente in der Naturanschauung von Bernard Palissy und Jacob Böhme », dans Greyerz K. von (dir.), Religion und Naturwissenschaften im 16. und 17. Jahrhundert, Gütersloher Verlaghaus, Güthersloh, p. 215-234.

Lees-Jeffries H., 2010, "An Elizabethan translation of Bernard Palissy's On Waters and Fountains", Studies in the History of Gardens and Designed lanscapes, vol. $30, \mathrm{n}^{\circ} 1$, p. 1-56.

Lestringant F., 2012, «Bernard Palissy oder die Unheimlichkeit. Der 'königliche Töpfermeister' und seine

Renaissance ", dans Lestringant F., Die Erfindung des Raums : Kartographie, Fiktion und Alterität in der Literatur der Renaissance. Erfurter MercatorVorlesungen, Transcript Verlag, Bielefeld, p. 145-164

Luzzini F., 2014, « I mirabili di Monsieur Palissy ", Acque Sotterranee, Italian journal of groundwater, vol. 3, n 3, p. 83-84.
Newman W. R., 2014, « Gotteshandwerk: Nachahmung und Neuschöpfung der Natur ", dans Cat. Exp. Düsseldorf, 2014 : Kunst und Alchemie, das Geheimnis der Verwandlung [Exp. Düsseldorf, Kunspalast, 2014], Hirmer, München, p. 116-122.

Palissy B., 2010, Euvres complètes, Fragonard M.-M. (dir.), seconde édition revue et annotée, Honoré Champion, Paris.

Pic M., 2012, «Un céramiste de légende : Palissy à Sèvres ", Sèvres, n 21, p. 31-45.

Pichon L., Calligaro T., Lemasson Q. et al., 2015, "Programs for visualization, handling and quantification of PIXE maps at the AGLAE facility", Nuclear Instruments and Methods in Physics Research, Section B : Beam interactions with materials and atoms, vol. 363, p. 48-54.

Plaziat J.-C., 2010, « Bernard Palissy (15101590), un authentique géologue ? Près de 500 ans d'incompréhension, entre oublis et réhabilitation au premier rang des précurseurs de la géologie ", Géochronique, n 115 , p. 11-15.

Plaziat J.-C., 2010, « Bernard Palissy (1510$1590)$, près de trois siècles de malentendus de la part des géologues, qu'il convient de dissiper à l'occasion de son $500^{\mathrm{e}}$ anniversaire ", Travaux $d u$ Comité français d'histoire de la géologie, COFRHIGEO, $3^{\mathrm{e}}$ série, tome 24, p. 131151.

Plaziat J.-C., 2011, "Bernard Palissy (15101590) and the French geologists: a critical reappraisal concerning the founding naturalist and his rustic ceramics", Bulletin de la société géologique de France, vol. 182, $\mathrm{n}^{\circ} 3$, p. 255-267.

Roisine G., Capobianco N., Caurant D. et al., 2017, "The art of Bernard Palissy (1510$1590)$, influence of firing conditions on the microstructure of iron-coloured high-lead glazes", Applied Physics A, vol. $123, n^{\circ} 8$.

Salmon-Dalas, M., 2016, «Bernard Palissy : à la recherche des secrets de la nature ", Le Festin, n ${ }^{\circ}$ 97, p. 42-47.

Sartori É., 2010, «Bernard Palissy défenseur du savoir artisanal », Pour la science, mai 2010, n 391, p. 82-86.
Schmeisser M., 2011, «Erdgeschichte und Paläontologie im 17. Jahrhunder : Bernard Palissy, Agostino Scilla, Nicolaus Steno und Leibniz », dans Jaumann H., Diskurse der Gelehrtenkultur in der Frühen Neuzeit: ein Handbuch, De Gruyter, Berlin, p. 809-858.

Smith P. H., Beentjes T., 2010, "Nature and Art, Making and Knowing: Reconstructing Sixteenth-Century Life Casting Techniques", Renaissance Quarterly, vol. 63, n 1, p. 128-179.

Stoll M., 2011, “'Sagacious' Bernard Palissy: Pinchot, Marsh, and the Connecticut Origins of American Conservation", Environmental History, vol. 16, $\mathrm{n}^{\circ} 1$, p. 4-37.

Sylvos F., 2011, «Balzac et Bernard Palissy », L'année balzacienne, $\mathrm{n}^{\circ} 12$, p. 175-198.

Theuriau F.-G., 2011, La Grotte rustique de Bernard Palissy : le projet inachevé d'Écouen, Éditions universitaires européennes, Sarrebruck.

Viennet C., Starosta P. (photographe), 2010, Bernard Palissy et ses suiveurs du XVI siècle à nos jours : hymne à la nature, Faton, Dijon.

Vignon C., 2015, "Rare Saint-Porchaire ewer newly attributed to the celebrated ceramist Bernard Palissy", Frick Collection members' magazine, vol. $15, \mathrm{n}^{\circ} 2$, p. 2-5.

\section{Documents inédits}

Ferdinand J., Frommel S., Aikema B. (dir.), 2014, Artigiano delle riforme: stile rustico e ricerca della sapienza nell'opera di Bernard Palissy $(1510-1590)=$ Artisan des réformes: style rustique et recherche de la sapience dans l'œuvre de Bernard Palissy (15101590), thèse, EPHE, Paris, Università degli studi, Vérone.

Hunter S. J., 2017, The Rustiques Figulines of Bernard Palissy, Qualifying paper (Master of Arts), The Bard Graduate Center, Decorative Arts, Design History, Material Culture, New York.

Roisine G. ; Bouquillon A., Caurant D. (dir.), 2008, Céramiques glaçurées de Bernard Palissy : à la recherche des secrets d'un maître de la Renaissance, thèse, Paris sciences et lettres.

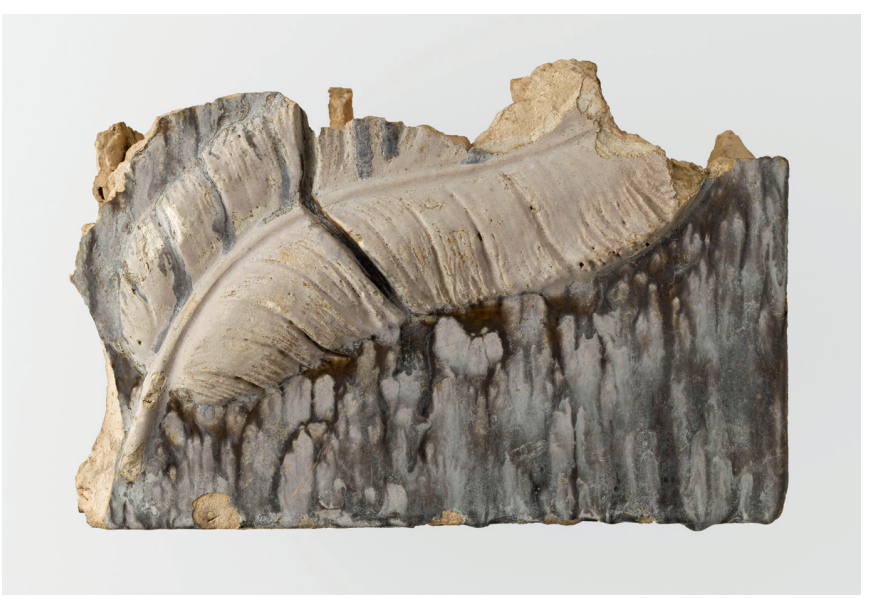

Fig. 1. Atelier de Bernard Palissy, Paris, fouilles des Tuileries, troisième quart du $\mathrm{XvI}^{\mathrm{e}}$ siècle, Brique : décor de plume, terre cuite glaçurée (L. 24,7 cm, 1. 16,5 cm, Pr. 14,8 cm), Écouen, musée national de la Renaissance, EP 435. (c) RMN-Grand Palais (musée national de la Renaissance, château d'Écouen)/ Stéphane Maréchalle. 